\title{
DESEMPENHO DE HÍBRIDOS DE MILHO NA REGIÃO MEIO-NORTE DO BRASIL
}

\author{
MILTON JOSÉ CARDOSO ${ }^{1}$, HÉLIO WILSON LEMOS DE CARVALHO ${ }^{2}$, MANOEL XAVIER DOS \\ SANTOS $^{3}$, MARIA DE LOURDES DA SILVA LEAL ${ }^{2}$, ANTÔNIO CARLOS DE OLIVEIRA ${ }^{3}$
}

${ }^{1}$ Eng. Agrôn., D.Sc., Embrapa Meio-Norte. Av. Duque de Caxias, 5650, Caixa Postal 01, CEP 64006-220 Teresina, PI. E-mail: milton@cpamn.embrapa.br (autor para correspondência).

${ }^{2}$ Eng. Agrôn., M.Sc., Embrapa Tabuleiros Costeiros. Av. Beira Mar, 3250, Caixa Postal 44, CEP 49025-040 Aracaju, SE. ${ }^{3}$ Eng. Agrôn., D.Sc., Embrapa Milho e Sorgo. Caixa Postal 151, CEP 35701-970 Sete Lagoas, MG.

\section{Revista Brasileira de Milho e Sorgo, v.2, n.1, p.43-52, 2003}

\begin{abstract}
RESUMO - Desenvolveu-se este trabalho com o objetivo de conhecer a adaptabilidade e a estabilidade de produção de diversos híbridos de milho, quando submetidos a diferentes condições ambientais na região Meio-Norte do Brasil. As produtividades médias alcançadas mostraram o grande potencial da região para a produção do milho, destacando-se como mais favoráveis ao cultivo do mesmo os municípios de São Raimundo das Mangabeiras, no Maranhão e, Baixa Grande do Ribeiro, Teresina e Parnaíba, no Piauí. A análise de variância conjunta mostrou diferenças significativas entre os híbridos para a produtividade de grãos e comportamento diferenciado frente às oscilações ambientais. Os híbridos que mostraram rendimentos médios acima da média geral $\left(7.166 \mathrm{~kg} \cdot \mathrm{ha}^{-1}\right)$ expressaram melhor adaptação, destacando-se, como genótipo ideal preconizado pelo modelo bissegmentado o híbrido simples A 2560. Os híbridos BRS 3060, Zeneca 84 E 90, Zeneca 8420, DKB 350, Zeneca 85 E 03 e Colorado 32 mostraram maior número de requisitos necessários para a adaptação a ambientes favoráveis. De especial interesse para a região também são os híbridos que evidenciaram boa adaptação (média superior á média geral), estimativas de $\mathrm{b}_{1}$ tendendo para zero e estimativas de $\mathrm{R}^{2}>80 \%$, a exemplo dos AG 6690, Dina 657, Agromen 3050, A 2366, AG 7575 e Agromen 2012.
\end{abstract}

Palavras-chave: Zea mays, cultivar, interação genótipo e ambientes, rendimento de grãos.

\section{HYBRID CORN PERFORMANCE IN MIDNORTHERN BRAZIL}

\begin{abstract}
This work is aimed at developing the adaptability and the production stability of the hybrid corn, when submitted to different environmental conditions in Mid-northern Brazil. The medium productivity showed the great potential of the region for the production of this corn, namely São Raimundo das Mangabeiras in Maranhão and Baixa Grande do Ribeiro, Teresina and Parnaiba in Piaui. The combined analysis variance showed significant differences among the hybrid for the productivity of grains and differentiated behavior due to environmental oscillations. The hybrid that showed medium yield above general average (7,166 kg.ha-1), adapted better, mainly the hybrid A 2560 for the bi-segmented model. The hybrids BRS 3060 , Zeneca 84 E 90, Zeneca 8420, DKB 350, Zeneca 85 E 03 and Colorado 32 showed a larger number of necessary requirements for the adaptation in the favorable environments. Special interest also for the hybrids that evidenced good adaptation (average higher than the general average), estimates of $b_{1}$ tending towards zero and estimates of $R^{2}>80 \%$, like the AG 6690 , Dina 657, Agromen 3050, A 2366, AG 7575 and Agromen 2012.
\end{abstract}

Key words: Zea mays, cultivar, interaction environment $\mathrm{x}$ genotype, grain yield. 
As condições de solo e clima prevalecentes na região Meio-Norte do Brasil permitem, com sucesso, a exploração da cultura do milho, que atualmente ocupa uma das maiores áreas plantadas naquela região. Apesar dos freqüentes problemas de irregularidade climática e do baixo nível tecnológico, a produtividade é uma das mais altas do Nordeste brasileiro (1.100 kg.ha ${ }^{-1}$ ) (Embrapa Meio-Norte, 2000). O consumo de milho nessa região é crescente, tendo sido registrado, no ano de 2000, déficit de 760.000 toneladas e 366.000 toneladas, respectivamente, nos Estados do Piauí e Maranhão. Dessa forma, faz-se necessária a importação de grande quantidade desses produto para suprir a demanda estadual, a qual vem se elevando, devido ao aumento significativo da avicultura e suinocultura. A região, contudo, dispõe ainda de uma extensa fronteira agrícola favorável à expansão da área cultivada e ao aumento da produtividade, localizada nas áreas de cerrado no Sul do Estado do Maranhão e no Sudoeste piauiense. $\mathrm{O}$ aumento do interesse pelo cultivo de milho nessas áreas deu-se em função da facilidade de mecanização e do desenvolvimento de sistemas de produção mais eficientes, fazendo com que a cultura se transformasse em uma atividade de cunho empresarial.

Sabe-se que o incremento da produtividade envolve diversos fatores, destacando-se, entre eles, a escolha correta de uma cultivar para exploração em uma determinada região. Anualmente, diversos híbridos de milho vêm sendo lançados no mercado, por empresas oficiais e privadas, havendo necessidade de avaliá-los em uma grande amplitude de condições ambientais e com a maior previsão possível, visando identificar aqueles de maior potencial produtivo nas condições ambientais da região.

Na região Meio-Norte do Brasil, o programa de avaliação de híbridos de milho vem sendo realizado em diferentes ambientes, esperando-se, nessas condições, a ocorrência de interação genótipos e ambientes. Cardoso et al. (1997 e 2000) detectaram a presença dessa interação em ensaios de competição de variedades e híbridos, conduzidos na região Meio-Norte do Brasil, ressaltando que, para tornar a recomendação de cultivares a mais segura possível, foi necessário identificar aquelas de melhor adaptabilidade e estabilidade de produção (Ramalho et al., 1993). Procedimentos semelhantes foram adaptados em diversas oportunidades, em outros locais da região Meio-Norte, por Cardoso et al. (1997; 2000), e do Nordeste brasileiro, por Carvalho et al. (1999; 2000 a; 2001). De forma semelhante, Arias (1996), no Estado do Mato Grosso, Carneiro (1998), no Estado do Paraná, e Ribeiro et al. (1999), em Minas Gerais, fizeram a recomendação mais segura de cultivares, após o estudo da adaptabilidade e estabilidade de produção. Neste contexto, há na literatura diversos metódos que podem ser utilizados (Eberhart \& Russel, 1966; Silva \& Barreto, 1985; Lin \& Binns, 1988; Cruz et al., 1989; Annicchiarico, 1992), os quais diferem nas estimativas dos parâmetros de adaptabilidade e estabilidade e na interpretação dos resultados.

Considerando-se esses aspectos, desenvolveu-se este trabalho objetivando conhecer a adaptabilidade e a estabilidade de produção de diversos híbridos de milho, quando submetidos a diferentes condições ambientais na região Meio-Norte do Brasil, para fins de recomendação.

\section{Material e Métodos}

Foram utilizados os dados de produtividade de grãos $\left(\mathrm{kg} \cdot \mathrm{ha}^{-1}\right)$ de onze ensaios de competição de híbridos de milho, executados na região MeioNorte do Brasil, no ano agrícola de 2000/2001, distribuídos nos Estados do Maranhão (quatro ensaios) e Piauí (sete ensaios), sendo dois ensaios do Estado do Piauí executados sob regime de irrigação 
por aspersão convencional. Os ambientes mostraram diferentes regimes pluviométricos (Tabela 1) e encontram-se localizados entre os paralelos $2^{\circ} 63^{\prime} \mathrm{S}$, em Parnaíba, PI, a 94'S, em Bom Jesus, PI, englobando diferentes condições ambientais (Tabela 2).

Utilizou-se o delineamento experimental em blocos ao acaso, com três repetições dos quarenta e um híbridos (Tabela 3). Cada parcela constou de quatro fileiras de 5,0 $\mathrm{m}$ de comprimento, a espaços de $0,80 \mathrm{~m}$ e $0,50 \mathrm{~m}$ entre covas, dentro das fileiras. Foram colocadas três sementes por cova, deixando-se, após o desbaste, duas plantas por cova. As adubações de cada ensaio obedeceram aos resultados das análises de solo de cada área experimental

TABELA 1. Coordenadas geográficas dos locais e tipos de solo das áreas experimentais.

\begin{tabular}{llrccc}
\hline Estado & Município & Latitude(S) & Longitude(W) & Altitude (m) & Tipos de solo ${ }^{(1)}$ \\
\hline Maranhão & S.R. das & $7^{\circ} 22^{\prime}$ & $45^{\circ} 36^{\prime}$ & 225 & AVA \\
& Mangabeiras & $7^{\circ} 08^{\prime}$ & $45^{\circ} 20^{\prime}$ & 212 & AA \\
& Sambaíba & $5^{\circ} 43^{\prime}$ & $45^{\circ} 18^{\prime}$ & 84 & LA \\
& Barra do Corda & $3^{\circ} 41^{\prime}$ & $42^{\circ} 45^{\prime}$ & 55 & LA \\
& Brejo & $5^{\circ} 05^{\prime}$ & $42^{\circ} 49^{\prime}$ & 72 & NF \\
Piauí & Teresina & $2^{\circ} 53^{\prime}$ & $41^{\circ} 41^{\prime}$ & 15 & NQ \\
& Parnaíba & $8^{\circ} 43^{\prime}$ & $44^{\circ} 14^{\prime}$ & 270 & LA \\
& Palmeiras do & $9^{\circ} 04^{\prime}$ & $44^{\circ} 21^{\prime}$ & 277 & LA \\
& Piaui & $7^{\circ} 32^{\prime}$ & $45^{\circ} 14^{\prime}$ & 325 & AA \\
\hline
\end{tabular}

(1) $\mathrm{AVA}=$ Argissolo Vermelho Amarelo, $\mathrm{AA}=$ Argissolo Amarelo, $\mathrm{LA}=$ Latossolo Amarelo, $\mathrm{NF}=$ Neossolo Flúvico, $\mathrm{NQ}=$ Neossolo Quartzarênico.

TABELA 2. Índices pluviais ( $\mathrm{mm}$ ) ocorridos durante o período experimental. Região Meio-Norte do Brasil, 2000-2001.

\begin{tabular}{lccccccc}
\hline Locais & 2000 & \multicolumn{3}{c}{2001} & \multirow{2}{*}{ Total } \\
\hline & Dez. & Jan. & Fev. & Mar. & Abr. & Mai. & \\
\cline { 2 - 6 } S.R.das Mangabeiras & $369,0^{(1)}$ & 136,0 & 80,5 & 177,5 & 103,0 & 28,0 & 894,0 \\
Sambaíba & $429,0^{(1)}$ & 126,0 & 249,0 & 293,0 & 127,0 & 28,0 & 1252,0 \\
Barra do Corda & - & $108,2^{(1)}$ & 48,8 & 102,5 & 96,6 & 17,8 & 474,9 \\
Brejo & - & $190,0^{(1)}$ & 213,0 & 536,0 & 65,0 & 96,0 & 1100,0 \\
Teresina & - & $253,6^{(1)}$ & 239,8 & 244,1 & 312,5 & 18,3 & 1068,3 \\
Parnaíba & - & $175,2^{(1)}$ & 245,2 & 119,1 & 379,2 & 46,3 & 965,0 \\
Palmeiras do Piauí & $314,5^{(1)}$ & 123,0 & 184,0 & 135,0 & 128,0 & 56,5 & 941,0 \\
Bom Jesus & $426,0^{(1)}$ & 190,0 & 161,0 & 327,0 & 129,0 & 73,0 & 1240,0 \\
Baixa G. do Ribeiro & $388,9^{(1)}$ & 154,3 & 278,9 & 222,4 & 148,2 & 42,7 & 1235,4 \\
\hline
\end{tabular}

(1) Mês de plantio dos experimentos. 
TABELA 3. Características dos híbridos avaliados na região Meio-Norte do Brasil. Ano agrícola de 2000/2001.

\begin{tabular}{|c|c|c|c|c|c|}
\hline Híbridos & Tipo & Ciclo & Dureza do grão & Cor do grão & Empresa \\
\hline Zeneca 84 E 90 & Simples & Precoce & Duro & Alaranjado & Agrosciences \\
\hline Zeneca 8420 & Simples & Precoce & Duro & Alaranjado & Agrosciences \\
\hline Dina 657 & Simples & Precoce & Semiduro & Alaranjado & Agrosciences \\
\hline Zeneca 8460 & Simples & Precoce & Duro & Alaranjado & Agrosciences \\
\hline Zeneca 8410 & Simples & Precoce & Semiduro & Alaranjado & Agrosciences \\
\hline Zeneca 84 E 03 & Simples & Precoce & Duro & Alaranjado & Agrosciences \\
\hline Colorado 32 & Triplo & Precoce & Semiduro & Laranja & Agrosciences \\
\hline AG 1051 & Duplo & Semiprecoce & Dentado & Amarelo & Monsanto \\
\hline AG 6690 & Triplo & Precoce & Semiduro & Alaranjado & Monsanto \\
\hline Cargill 747 & Triplo & Precoce & Duro & Alaranjado & Monsanto \\
\hline DKB 350 & Triplo & Precoce & Semiduro & Alaranjado & Monsanto \\
\hline AG 7575 & Simples & Precoce & Duro & Avermelhado & Monsanto \\
\hline AG 8080 & Triplo & Precoce & Semiduro & Alaranjado & Monsanto \\
\hline AG 9010 & Simples & Superprecoce & Duro & Alaranjado & Monsanto \\
\hline Cargill 435 & Duplo & Precoce & Semiduro & Alaranjado & Monsanto \\
\hline Pioneer X $1318 \mathrm{H}$ & Simples & Precoce & Semiduro & Alaranjado & Pioneer \\
\hline Pioneer 3021 & Triplo & Precoce & Semiduro & Alaranjado & Pioneer \\
\hline Poneer $30 \mathrm{~K} 75$ & Simples & Precoce & Semiduro & Alaranjado & Pioneer \\
\hline Pioneer 30 F 88 & Simples & Precoce & Semiduro & Alaranjado & Pioneer \\
\hline Agromen 3050 & Simples & Superprecoce & Duro & Alaranjado & Agromen \\
\hline Agromen 2012 & Duplo & Superprecoce & Semiduro & Alaranjado & Agromen \\
\hline Agromen 3150 & Triplo & Superprecoce & Duro & Avermelhado & Agromen \\
\hline Agromen 3180 & Triplo & Precoce & Duro & Alaranjado & Agromen \\
\hline Agromen 3060 & Triplo & Superprecoce & Duro & Alaranjado & Agromen \\
\hline A 2366 & Simples & Precoce & Duro & Alaranjado & Bayer \\
\hline A 2560 & Simples & Precoce & Duro & Alaranjado & Bayer \\
\hline A 3565 & Triplo & Precoce & Duro & Alaranjado & Bayer \\
\hline A 3663 & Triplo & Precoce & Semiduro & Alaranjado & Bayer \\
\hline A 2288 & Simples & Precoce & Duro & Alaranjado & Bayer \\
\hline A 2005 & Simples & Superprecoce & Duro & Alaranjado & Bayer \\
\hline BRS 3060 & Triplo & Superprecoce & Semidentado & Alaranjado & Embrapa \\
\hline BR 3123 & Triplo & Precoce & Semiduro & Alaranjado & Embrapa \\
\hline BR 206 & Duplo & Precoce & Semidentado & Alaranjado & Embrapa \\
\hline HT 1 & Triplo & Precoce & Semidentado & Alaranjado & Embrapa \\
\hline BRS 3101 & Triplo & Precoce & Semiduro & Alaranjado & Embrapa \\
\hline HT 5 & Triplo & Precoce & Semiduro & Alaranjado & Embrapa \\
\hline BRS 2110 & Duplo & Precoce & Semidentado & Alaranjado & Embrapa \\
\hline SHS 5050 & Triplo & Superprecoce & Semidentado & Alaranjado & Santa Helena \\
\hline SHS 5070 & Triplo & Superprecoce & Semidentado & Alaranjado & Santa Helena \\
\hline MR 2601 & Simples & Precoce & Semiduro & Alaranjado & Bayer \\
\hline
\end{tabular}


e da exigência da cultura. Foram colhidas as duas fileiras centrais de forma integral, correspondendo a uma área útil de $8,0 \mathrm{~m}^{2}$.

Os dados de produtividade de grãos foram submetidos a uma análise de variância por ambiente, obedecendo ao modelo em blocos ao acaso, e a uma análise de variância conjunta, obedecendo-se ao critério de homogeneidade dos quadrados médios residuais, considerando-se aleatórios os efeitos de blocos e ambientes e, fixo o efeito de cultivares. Os parâmetros de adaptabilidade e estabilidade foram estimados segundo a metodologia proposta por Cruz et al. (1989), que utiliza o seguinte modelo: $\mathrm{Y}_{\mathrm{ij}}=\mathrm{b}_{\mathrm{oi}}+\mathrm{b}_{1 \mathrm{i}} \mathrm{I}_{\mathrm{J}}+\mathrm{b}_{2 \mathrm{i}} \mathrm{T}\left(\mathrm{I}_{\mathrm{j}}\right)+\sigma_{\mathrm{ij}}+\mathrm{e}_{\mathrm{ij}}$, em que: $\mathrm{Y}_{\mathrm{ij}}$ média da cultivar i no ambiente $\mathrm{j}$; $\mathrm{I}_{\mathrm{j}}$ : índice ambiental; T $\left(I_{j}\right)=0$ se $I_{J}<0 ; T\left(I_{J}\right)=I_{j}-I_{+}$se $I_{j}>0$, sendo $I_{+}$a média dos índices $I_{j}$ positivos; $b_{0 i}:$ média geral da cultivar $i ; b_{1 i}$ : coeficiente de regressão linear associado à variável $\mathrm{I}_{\mathrm{j}} ; \mathrm{b}_{2 \mathrm{i}}$ : coeficiente de regressão linear associado à variável $\mathrm{T}\left(\mathrm{I}_{\mathrm{j}}\right) ; \sigma_{\mathrm{ji}}$ : desvio da regressão linear; $\mathrm{e}_{\mathrm{ij}}$ : erro médio experimental.

\section{Resultados e Discussão}

Foram encontradas diferenças significativas entre os híbridos, nas análises de variância por ambiente. A média de produtividade nos ensaios variou de $5.481 \mathrm{~kg} \cdot \mathrm{ha}^{-1}$, no município de Palmeiras do Piauí, PI, a $8.368 \mathrm{~kg} \cdot \mathrm{ha}^{-1}$, no município de São Raimundo das Mangabeiras, MA(Tabela 4), o que evidencia uma ampla faixa de variação nas condições ambientais em que foram realizados os ensaios. Os municípios de São Raimundo das Mangabeiras, MA, e Teresina e Parnaíba, tanto em regime de sequeiro quanto sob irrigação, e Baixa Grande do Ribeiro (sequeiro), PI, apresentaram as melhores potencialidades para o desenvolvimento do milho, com produtividades médias entre $7.498 \mathrm{~kg}^{-h^{-1}} \mathrm{e}$ $8.368 \mathrm{~kg} \cdot \mathrm{ha}^{-1}$, destacando-se como ambientes mais favoráveis para a exploração do milho. Os município de Barra do Corda, MA, e Bom Jesus, PI, também apresentam boa potencialidade para o cultivo do milho. Os coeficientes de variação obtidos oscilaram de 7\% a 12\% (Tabela 4), conferindo boa precisão aos ensaios, conforme critérios adotados por Scapin et al. (1995).

TABELA 4. Produtividades médias de grãos e coeficientes de variação obtidos nos onze ambientes da região Meio-Norte do Brasil, no ano agrícola de 2000/2001.

\begin{tabular}{llcc}
\hline Ambiente & & $\begin{array}{c}\text { Produtividade média } \\
\left(\mathrm{kg} \cdot \mathrm{ha}^{-1}\right)\end{array}$ & $\begin{array}{c}\text { Coeficiente de variação } \\
(\%)\end{array}$ \\
\hline Maranhão & Sambaíba & 5.867 & 12 \\
& S.R. das Mangabeiras & 8.368 & 9 \\
& Brejo & 5.579 & 9 \\
& Barra do Corda & 6.830 & 10 \\
Piauí & Teresina sequeiro & 8.136 & 8 \\
& Parnaíba sequeiro & 8.010 & 7 \\
& Palmeiras do Piauí & 5.481 & 11 \\
& Bom Jesus & 6.524 & 10 \\
& Baixa G. do Ribeiro & 8.086 & 7 \\
& Teresina irrigado & 7.498 & 7 \\
\hline
\end{tabular}


Verificaram-se diferenças significativas para o efeito de ambientes, híbridos e interação híbridos e ambientes (Tabela 5). A presença da interação evidencia um comportamento diferenciado dos híbridos nos diferentes ambientes, permitindo-se, assim, o estudo pela análise de estabilidade proposta.

As estimativas dos parâmetros de adaptabilidade e estabilidade estão na Tabela 6 , verificando-se que as produtividades médias de grãos $b_{0}$ variaram de $6.261 \mathrm{~kg}^{-h^{-1}}$ (BRS 2110) a 8.009 kg.ha-1 (Zeneca 84 E 90), com média geral de 7.116 $\mathrm{kg} \cdot \mathrm{ha}^{-1}$, o que denota o alto potencial para a produtividade dos híbridos avaliados. Aliado ao modelo proposto, consideram-se como genótipos melhores adaptados aqueles que expressaram rendimentos médios de grãos superiores à média geral (Vencovsky \& Barriga, 1992).

Quanto ao coeficiente de regressão $\left(b_{1}\right)$, que corresponde à resposta linear do material a variações ambientais desfavoráveis, verificou-se que, no grupo de híbridos que mostrou melhor adaptação (rendimentos médios acima da média geral), os Zeneca 84 E 90, AG 1051, Zeneca 8420, DKB 350, Pioneer 3021, Zeneca 85 E 03 e Colorado 32 mostraram-se exigentes nas condições desfavoráveis $\left(b_{1}>1\right)$, enquanto que os BRS 3060 e A 2560 foram mais estáveis nessas condições $\left(b_{1}<1\right)$. A estimativa $b_{1}+b_{2}$, que avalia o desempenho dos materiais nos ambientes favoráveis, mostrou que, entre os híbridos de melhor adaptação, apenas o Zeneca 84 E 60, A 2366, BRS 3060 e A 2560 responderam à melhoria ambiental $\left(b_{1}+b_{2}>1\right)$.

A maioria das cultivares avaliadas mostrou os desvios da regressão estatisticamente diferente de zero (Tabela 6), o que, pelo método proposto, indica comportamento imprevisível nos ambientes considerados. Dessa forma, observa-se que, no grupo de híbridos que expressou melhor adaptação $\left(b_{0}>\right.$ média geral), apenas o Zeneca 84E90, AG 6690, DKB 350, AG 7575, Agromen 2012 e Zeneca 85 E 03 mostraram melhor estabilidade nos ambientes considerados $\left(\mathrm{s}^{2} \mathrm{~d}=0\right)$. Apesar disso, Cruz et al. (1989) enfatizaram que a estabilidade pode ser avaliada pela estimativa de $\mathrm{R}^{2}$ e que aqueles materiais que apresentaram estimativas de $\mathrm{R}^{2}$ acima de $80 \%$ não devem ter os seus graus de previsibilidade comprometidos. Constatou-se, também, que o nível de estabilidade observada nos híbridos independeu de suas respectivas bases genéticas, podendo-se, identificar híbridos de diferentes níveis de estabilidade dentro de qualquer grupo (simples, triplo ou duplos), havendo boa concordância com o relatado por Cardoso et al. (2000) e por Carvalho et al. (1999, 2000a, 2000b, e 2001). De fato, observa-se que os melhores níveis de estabilidade foram registrados nos híbridos simples Zeneca 84 E 90, Zeneca 8420, Agromen 3050, AG 7575 e Zeneca 85 E 03, nos híbridos triplos AG 6690 e DKB 350 e duplo

TABELA 5. Análise da variância conjunta da produtividade de grãos de 41 híbridos de milho, em 11 ambientes da região Meio-Norte do Brasil, no ano agrícola de 2001.

\begin{tabular}{lcc}
\hline Fonte de variação & Graus de liberdade & Quadrados Médios \\
\hline Ambientes (A) & 10 & $150831236,0^{* * *}$ \\
Híbridos (H) & 40 & $5539600,9^{* *}$ \\
Interação $(\mathrm{A}$ x H) & 400 & $1177077,0^{* * *}$ \\
Resíduo Médio & 880 & - \\
\hline Média $\left(\mathrm{kg}^{-1}\right.$ ha $\left.^{-1}\right)$ & & 7.116 \\
CV $(\%)$ & & 8,6 \\
\hline
\end{tabular}

**Significativo a $1 \%$ de probabilidade, pelo teste $\mathrm{F}$. 
TABELA 6. Produtividade média de grãos $\left(\mathrm{kg} \cdot \mathrm{ha}^{-1}\right)$ e estimativas dos parâmetros de adaptabilidade e estabilidade de 41 híbridos de milho, em 11 ambientes da região Meio-Norte do Brasil, no ano agrícola de 2000/ 2001. Modelo Cruz et al. (1989).

\begin{tabular}{|c|c|c|c|c|c|c|c|c|}
\hline \multirow{2}{*}{ Hibridos } & \multicolumn{3}{|c|}{ Médias nos ambientes } & \multirow{2}{*}{$\mathbf{b}_{1}$} & \multirow{2}{*}{$\mathrm{b}_{2}$} & \multirow{2}{*}{$\mathbf{b}_{1}+\mathbf{b}_{2}$} & \multirow{2}{*}{$\mathrm{S}$} & \multirow{2}{*}{$\begin{array}{l}\mathrm{R}^{2} \\
(\%)\end{array}$} \\
\hline & Geral & Desfavorável & Favorável & & & & & \\
\hline Zeneca 84 E $90^{\top}$ & 8.009 & 6.338 & 9.402 & $1,48^{* 4}$ & $-1,11$ & 0,35 & $598177,3 \mathrm{~ns}$ & 94 \\
\hline $\mathrm{AG} 1051^{3}$ & 8.005 & 8.496 & 9.262 & $1,31^{* 4}$ & 0,64 & 1,96 & $2619967,7^{44}$ & 76 \\
\hline Pioneer X $1318 \mathrm{H}^{1}$ & 7.758 & 6.837 & 8.526 & $0,99 \mathrm{~ns}$ & $-1,25$ & $=0.26$ & $2367656,9^{* 6}$ & 64 \\
\hline Zeneca $8420^{1}$ & 7.613 & 6.230 & 8.765 & $1,24^{*}$ & $-0,83$ & 0,41 & $743940,2^{*}$ & 60 \\
\hline $\mathrm{AG} 6690^{2}$ & 7.613 & 6.332 & 8.680 & 1,15 & 0,11 & 1,26 & $487461,5 \mathrm{~ns}$ & 92 \\
\hline Dina $657^{1}$ & 7.495 & 6.276 & 8.511 & 1,05 & $-0,56$ & 0,49 & $1057315,5^{* *}$ & 83 \\
\hline Zeneca 84 E $60^{7}$ & 7.483 & 6.081 & 8.651 & 1,18 & $1.52^{4 *}$ & $2,71^{* 4}$ & $1847052,1^{44}$ & 80 \\
\hline Cargill $747^{\frac{1}{3}}$ & 7.472 & 6.620 & 8.182 & 0,81 & 0,30 & 1,12 & $1587286,9^{* 4}$ & 66 \\
\hline DKB $350^{2}$ & 7.459 & 6.202 & 8.507 & $1,21^{*}$ & 0,38 & 1,60 & $166137, \operatorname{lns}$ & 98 \\
\hline Agromen $3050^{1}$ & 7.448 & 6.430 & 8.290 & 0,95 & $-0,16$ & 0,80 & $416141,2 \mathrm{~ns}$ & 91 \\
\hline Pioneer $3021^{2}$ & 7.431 & 6.193 & 8.463 & $1,23^{*}$ & $-0,25$ & 0,98 & $2029340,6^{* *}$ & 77 \\
\hline A $2366^{1}$ & 7.334 & 6.277 & 8.325 & 0.92 & $1.57^{4 *}$ & $2,49^{* 4}$ & $866441,9^{*}$ & 84 \\
\hline $\mathrm{AG} 7575^{1}$ & 7.371 & 6.280 & 8.280 & 1,05 & 0,14 & 1,20 & $484882,6 \mathrm{~ns}$ & 91 \\
\hline BRS $3060^{2}$ & 7.293 & 6.565 & 7.900 & $0,80^{\circ}$ & $2,34^{* *}$ & $3,14^{* \hbar}$ & $1190401,1^{* 6}$ & 78 \\
\hline Zeneca $8410^{1}$ & 7.284 & 6.136 & 8.241 & 1,09 & 0,23 & 1,33 & $2182328,3^{* *}$ & 72 \\
\hline Agromen $2012^{3}$ & 7,267 & 5.919 & 8.390 & 1,16 & 0,11 & 1,28 & $489443,2 \mathrm{~ns}$ & 93 \\
\hline Zeneca 85 E 031 & 7.266 & 5.970 & 8.344 & $1,22^{*}$ & $-1,47^{*}$ & $-0,25^{* *}$ & $656377,8 \mathrm{~ns}$ & 91 \\
\hline A $2560^{1}$ & 7.257 & 6.375 & 7.992 & $0,77^{4}$ & $3,44^{4 *}$ & $4,21^{* 4}$ & $833751,4^{*}$ & 86 \\
\hline Pioneer 30 F $75^{1}$ & 7.224 & 5.975 & 8.264 & 1,17 & $-0,49$ & 0,68 & $1980498,1^{44}$ & 76 \\
\hline Colorado $32^{2}$ & 7.216 & 6.076 & 8.166 & $1,21^{*}$ & $-1,13^{*}$ & $=0,15^{\circ}$ & $1289194,3^{* 6}$ & 84 \\
\hline AG $8080^{2}$ & 7.114 & 5.759 & 8.244 & $1,31 * *$ & 0,70 & 2,01 & $845667,5^{*}$ & 91 \\
\hline SHS $5050^{2}$ & 7.008 & 5.854 & 7.971 & 1,14 & $-1,52$ & $-0,37^{* *}$ & $1564886,8 * *$ & 79 \\
\hline BR $3123^{2}$ & 7.000 & 6.245 & 7.630 & $0,78^{*}$ & 0,70 & 1,48 & $2127548,0^{* *}$ & 59 \\
\hline MR $2601^{1}$ & 6.973 & 5.996 & 7.788 & 0,93 & $-0,16$ & 0,77 & $1475605,0^{* *}$ & 73 \\
\hline SHS $5070^{2}$ & 6.968 & 5.991 & 7.782 & 0,90 & 0,23 & 1,13 & $823427,3^{*}$ & 82 \\
\hline BR $206^{3}$ & 6.944 & 5.963 & 7.762 & 0,90 & $-0,54$ & 0,35 & $414804,2 \mathrm{~ns}$ & 90 \\
\hline $\mathrm{HT}^{2}$ & 6.871 & 5.895 & 7.683 & 0.84 & $=0,60$ & 0.23 & $955362.8^{\text {** }}$ & 77 \\
\hline Pioneer $30 \mathrm{~F} 88^{1}$ & 6.861 & 5.566 & 7.940 & $1,21^{*}$ & $-0,49$ & 0,71 & $449174,8 \mathrm{~ns}$ & 94 \\
\hline BRS $3101^{2}$ & 6.834 & 6.021 & 7.512 & $0,75^{*}$ & 0,89 & 1,64 & $944631,8^{*}$ & 75 \\
\hline DAS $112 \mathrm{X}^{1}$ & 6.822 & 5.800 & 7.673 & 1,02 & $-1,20^{*}$ & $-0,18^{*}$ & $843608,7^{*}$ & 85 \\
\hline $\mathrm{HT} 5^{2}$ & 6.791 & 6.077 & 7.386 & $0,70^{44}$ & $-0,32$ & 0,38 & $462503,9 \mathrm{~ns}$ & 83 \\
\hline AG $9010^{1}$ & 6.783 & 5.992 & 7.443 & 0,84 & $-0,99^{*}$ & $=0,14$ & $1100344,4^{\text {*t }}$ & 74 \\
\hline A $3565^{2}$ & 6.771 & 5.805 & 7.576 & 0,90 & $=0,19$ & 0,70 & $756316,6^{*}$ & 83 \\
\hline A $3663^{2}$ & 6.752 & 6.123 & 7.276 & $0,60^{* *}$ & 0,25 & 0,86 & $1487452,1^{* *}$ & 53 \\
\hline Agromen $3150^{2}$ & 6.680 & 5.647 & 7.541 & 0,89 & $-0,48$ & 0,41 & $392095,5 \mathrm{~ns}$ & 90 \\
\hline Cargill $435^{3}$ & 6.677 & 5.523 & 7.638 & 1,08 & 0,36 & 1,45 & $620308,1 \mathrm{~ns}$ & 90 \\
\hline Agromen $3180^{2}$ & 6.648 & 5.673 & 7.461 & 0.90 & 0,35 & 1,25 & $350764.9 \mathrm{~ns}$ & 92 \\
\hline A $2288^{1}$ & 6.619 & 5.621 & 7.500 & 0,85 & 0,56 & 1,41 & $1314485,5^{* 6}$ & 73 \\
\hline Agromen $3060^{2}$ & 6.554 & 5.647 & 7,310 & 0,83 & 0,26 & 1,09 & $531283,3 \mathrm{~ns}$ & 86 \\
\hline A $2005^{1}$ & 6.446 & 5.078 & 7,586 & 1,13 & $-1,82^{* *}$ & $-0,69$ & $2368647,5^{* *}$ & 71 \\
\hline BRS $2110^{2}$ & 6.261 & 5.946 & 6,523 & $0,37^{* *}$ & 0,71 & 1,08 & $716623,5^{*}$ & 52 \\
\hline Média $\left(\mathrm{kg}, \mathrm{ha}^{-1}\right)$ & 7.166 & & & & & & & \\
\hline C.V. $(\%)$ & 8,6 & & & & & & & \\
\hline D.M.S. $5 \%\left(\mathrm{~kg}^{\left.-\mathrm{ha}^{-1}\right)}\right.$ & 1.058 & & & & & & & \\
\hline
\end{tabular}

${ }^{1}$ Híbrido simples, ${ }^{2}$ híbrido triplo $\mathrm{e}^{3}$ híbrido duplo

** e* Significativamente diferente da unidade para $b_{1}$ e $b_{1}+b_{2}$ e diferente de zero para $b_{2}$ pelo teste $t$ de Student, e diferente de zero para o quadrado médio dos desvios da regressão $\left(\mathrm{s}^{2} \mathrm{~d}\right)$, pelo teste $\mathrm{F}$, a $5 \%$ e $1 \%$ de probabilidade, respectivamente. 
Agromen 2012, com estimativas de $\mathrm{R}^{2}$ oscilando entre $90 \%$ e $98 \%$. Menores estimativas de $\mathrm{R}^{2}$, expressando baixa previsibilidade nos ambientes considerados, nesse mesmo grupo de materiais $\left(b_{0}>\right.$ média geral), foram observadas nos híbridos simples Pioneer X 1318 H e Pioneer 30 F 75 e nos duplos AG 1051, Cargill 747 e Pioneer 3021. A ocorrência desses resultados contraditórios sugere a importância do estudo da interação cultivares e ambientes, visando fornecer mais subsídios e maior compreensão do comportamento de diferentes cultivares de milho quando submetidos a diferentes condições ambientais (Arias, 1996). Observaramse também valores de $\mathrm{R}^{2}$ de $52 \%$ (BRS 2110) e 53\% (A 3663), evidenciando que o modelo não está adequado para explicar o comportamento desses dois materiais.

Analisando-se os resultados apresentados, percebe-se que a cultivar ideal preconizada pelo modelo de Cruz et al. (1989), ou seja, aquela que apresenta uma média de produtividade alta $\left(\mathrm{b}_{0}>\right.$ média geral), $a$ de $b_{1}<1,0$ (menos exigentes nas condições desfavoráveis) e estimativa de $b_{1}+b_{2}>$ 1,0 (responsivo à melhoria ambiental) e desvio da regressão semelhantes a zero, não foi encontrada no conjunto avaliado. Nesse conjunto avaliado, tampouco foi encontrado qualquer híbrido com adaptação específica a ambiente favorável $\left(\mathrm{b}_{0}>\right.$ média geral, $b_{1}$ e $b_{1}+b_{2}>1$ e s ${ }^{2} d=0$ ), apesar de os híbridos Zeneca 84 E 90, DKB 350, Zeneca 85 E 03 e Colorado 32 apresentarem maior número de requesitos necessários para adaptação nessa classe de ambientes $\left(b_{0}>\right.$ média geral, $b_{1}>1, b_{1}+b_{2}=1$ e $\mathrm{s}^{2} \mathrm{~d}=0$ ). Os híbridos BRS 3060, AG 1051 e Pioneer 3021, apesar de expressarem baixa estabilidade nos ambientes considerados $\left(\mathrm{s}^{2} \mathrm{~d}\right.$ diferente de zero), podem também ser recomendados para as condições favoráveis $\left(b_{0}>\right.$ média geral, $b_{1}>1$ e $\left.b_{1}+b_{2}=1\right)$. Neste trabalho, não foi encontrado híbrido com adaptação específica às condições desfavoráveis $\left(b_{0}>\right.$ média geral, $b_{1}$ e $b_{1}+b_{2}>1$ e $\left.s^{2} d=0\right)$. De especial interesse para a região são também os híbridos que evidenciaram boa adaptação $\left(\mathrm{b}_{0}>\right.$ média geral), estimativas de $b_{1}$ tendendo para zero, a exemplo dos AG 6690, Dina 657, Agromen 3050, A 2366, AG 7575 e Agromen 2012. O híbrido Pioneer X $1318 \mathrm{H}$, apesar de mostrar baixa estabilidade nos ambientes considerados, apresentou melhor desempenho produtivo nas condições desfavoráveis e estimativa de $b_{1}=1$, justificando sua recomendação para as condições desfavoráveis. Comportamento semelhante foi observado no híbrido Cargill 747.

\section{Conclusões}

Os híbridos com rendimentos médios acima da média geral expressaram boa adaptação na região Meio-Norte do Brasil, constituindo-se em alternativas importantes para os sistemas de produção melhor tecnificados.

O híbrido A 2560 destacou-se como o genótipo ideal preconizado pelo modelo bissegmentado.

Não foi encontrado qualquer híbrido com adaptação específica a ambientes favoráveis. Os híbridos Zeneca 84 E 60, DKB 350, Zeneca 85 E 031 e Colorado 32 mostraram maior número de requisitos necessários para adaptação nessas condições de ambientes.

\section{Literatura Citada}

ANNICCHIARICO, P. Cultivar adaptation and recomendation from alfafa. Trials in Northern Italy. Journal os Genetic \& Berrding, Rome, v.46, n.3, p.269-278, 1992.

ARIAS, E. R. A. Adaptabilidade e estabilidade de cultivares de milho no Estado do Mato Grosso do Sul e avanço genético obtido no período de 1986/87 a 1993/94. 1996. 118f. Tese (Doutorado) - Escola Superior de Agricultura de Lavras, Lavras. 
CARDOSO, M.J.; CARVALHO, H.W.L. de; PACHECO, C. A. P.; SANTOS, M. X. dos; LEAL, M. de L. da S. Adaptabilidade e estabilidade de cultivares de milho no estado do Piauí, no biênio 1993/1994. Revista Científica Rural, Bagé, v.2, n.1, p.35-44, 1997.

CARDOSO, M. J.; CARVALHO, H. W. L. de.; LEAL, M. de L da S.; SANTOS, M X. dos. Comportamento, adaptabilidade e estabilidade de híbridos de milho no Estado do Piauí no ano agrícola de 1998. Revista Científica Rural, Bagé, v.5, n.1, p.146-153, 2000.

CARNEIRO, P. C. S. Novas metodologias de análise de adaptabilidade e estabilidade de comportamento. 1998. 168f. Tese (Doutorado) - Universidade Federal de Lavras, Lavras.

CARVALHO, H. W. L. de.; LEAL, M. de L da S.; CARDOSO, M. J.; SANTOS, M X. dos; CARVALHO, B. C. L. de.; TABOSA, J. N.; LIRA, M.A.; ALBUQUERQUE, M. M. Adaptabilidade e estabilidade de cultivares e híbridos de milho no Nordeste brasileiro no ano agrícola de 1998. Pesquisa Agropecuária Brasileira, Brasília, v.36, n.4, p.637-644, 2001.

CARVALHO, H. W. L. de.; LEAL, M. de L da S.; SANTOS, M X. dos.; CARDOSO, M. J.; MONTEIRO, A.A.T.; TABOSA, .Adaptabilidade e estabilidade de cultivares de milho no Nordeste brasileiro. Pesquisa Agropecuária Brasileira, Brasília, v.35, n.6, p.1115-1123, 2000b.

CARVALHO, H. W. L. de.; LEAL, M. de L da S.; SANTOS, MX. dos.; MONTEIRO,A.A.T.; CARDOSO, M. J.; CARVALHO, B. C. L. de. Estabilidade de cultivares de milho em três ecossistemas do Nordeste brasileiro. Pesquisa Agropecuária Brasileira, Brasília, v.35, n.9, p.1773-1781, 2000a.
CARVALHO, H. W. L. de.; SANTOS, M X. dos.; LEAL, M. de L da S. PACHECO, C. A. P; CARDOSO, M. J.; MONTEIRO A. A. T. Adaptabilidade e estabilidade de produção de cultivares de milho no Nordeste brasileiro. Pesquisa Agropecuária Brasileira, Brasília, v.34, n.9, p.1581-1591, 1999.

CRUZ, C. D.; TORRES, R. A. de.; VENCOVSKY,R. An alternative approach to the stability analisis by Silva and Barreto. Revista Brasileira de Genética, Ribeirão Preto, v. 12, p.567580, 1989.

EBERHART, S. A.; RUSSEL, W. A. Stability parameters for compaing varieties. Crop Science, Madison, v.6, n.1, p.36-40, 1966.

EMBRAPA MEIO-NORTE (Teresina PI). II Plano Diretor da Embrapa Meio-Norte 2000-2003. Teresina, 2000.35p.

LIN, C. S.; BINNS, M. R. A superiority measure of cultivar performance for cultivar $\mathrm{x}$ location data. Canadian Journal of Plant Science, Ottawa, v. 68, n. 1, p. 193-198, 1988.

RAMALHO, M A. P.; SANTOS, J. B. dos.; ZIMMERMANN, M. J de O. Genética quantitativa em plantas autógamas: aplicação no melhoramento do feijoeiro. Goiânia, Editora UFG, 1993. cap. 6, p.131-169. (Publicação, 120).

RIBEIRO, P. H. E.; RAMALHO, M, A. P.; FERREIRA, D. F. Adaptabilidade e estabilidade de cultivares de milho avaliadas em diferentes condições ambientais do Estado de Minas Gerais. In: REUNION LATINOAMERICANADEL MAIZ, 28, 1999, Sete Lagoas, Memórias...Sete Lagoas: Embrapa Milho e Sorgo/CIMMYT, 1999. p.251260.

SCAPIM, C. A.; CARVALHO, C. G. P de.; CRUZ, C. D. Uma proposta de classificação dos 
coeficientes de variação para a cultura do milho. AGRONÔMICA, 1, Piracicaba, 1985. Resumos Pesquisa Agropecuária Brasileira, Brasília, v30, ... Piracicaba, Fundação Cargill, 1985.p.49-50. n.5, p.683-686, 1995.

VENCOVSKY, R.; BARRIGA, P. Genética SILVA, J. G.; BARRETO, J. N. Aplicação da rebiométrica no fitomelhoramento. Ribeirão Preto: gressão linear segmentada em estudos da interação genótipo por ambiente. In: SIMPÓSIO DE ESTARibeirão Preto, Sociedade Brasileira de Genética, TÍSTICA APLICADA À EXPERIMENTAÇÃO 1992. 426p. 